\title{
A RIEMANN INTEGRAL IN A LOCALLY COMPACT HAUSDORFF SPACE
}

\author{
S. I. AHMED and W. F. PFEFFER
}

(Received 29 May 1984)

Communicated by $\mathbf{R}$. Vyborny

\begin{abstract}
We present a systematic and self-contained exposition of the generalized Riemann integral in a locally compact Hausdorff space, and we show that it is equivalent to the Perron and variational integrals. We also give a necessary and sufficient condition for its equivalence to the Lebesgue integral with respect to a suitably chosen measure.
\end{abstract}

1980 Mathematics subject classification (Amer. Math. Soc.): 28 A 25, 26 A 42; secondary 54 C 30, 28 C 05, 28 C 15.

\section{Introduction}

In this paper, we present a systematic, virtually self-contained and, in our opinion, fairly complete development of the Riemann integral in a locally compact Hausdorff space. Such an integral was first introduced by McShane (seek [3, Example 6]), who also investigated its relationship to the Lebesgue integral and gave sufficient conditions under which the integral depends only on the integrand restricted to the integration domain (rather than to the closure of it). We improve and unify the results of McShane by showing that the Riemann integral induces a regular measure, and that the Lebesgue integral with respect to this measure is equal to the Riemann integral if and only if the Riemann integral depends only on the integrand restricted to the integration domain.

We believe that the completeness of our presentation has a substantial merit in making McShane's idea accesible to a larger audience. Indeed in [3], the Riemann

This work originated from the Master's thesis of S. I. Ahmed written under the supervision of W. F. Pfeffer at the University of Petroleum and Minerals (UPM), Dhahran, Saudi Arabia. The work of W. F. Pfeffer was partially supported by UPM Research Project MATH/MANIFOLD/68.

(C) 1986 Australian Mathematical Society $0263-6115 / 86$ \$A2.00 +0.00 
integral we study is mentioned only briefly as an example of a more general theory, and it is difficult, if not impossible, to appreciate it fully without studying the whole memoir. There are various places in the literature where the Riemann integral of McShane is discussed in the real line or in a finite dimensional Euclidean space (see, e.g., [4], [2], and [9]). However, this is an unnecessarily restrictive setting in which some important points are left unnoticed: for example, the dependence of the integral on values the integrand takes outside the integration domain (see Example 7.5). To our knowledge, a sufficiently detailed treatment of the Riemann integral in a topological space has not been available.

The paper is organized as follows. After necessary preliminaries (Section 2), we define the Riemann integral of McShane and we derive for it all those properties which are considered standard for any integral worth the name (Sections 3-5). The essential equivalence of the Riemann and Lebesgue integrals is established in Sections 6 and 7. In Section 8, we show that the Riemann integral coincides with the Perron integral associated to the natural convergence (see [6, Section 2] and [7, Corollary 2.15]). We also show the equivalence of the Riemann integral and the variational integral of Henstock (see [1]).

\section{Preliminaries}

By $\mathbf{R}$ we denote the set of all real numbers. If $a, b \in \mathbf{R}$, we let $a \vee b=$ $\max \{a, b\}$ and $a \wedge b=\min \{a, b\}$.

If $S$ is a set, then $\mathscr{F}(S)$ is the family of all functions $f: S \rightarrow \mathbf{R}$. The algebraic operations, lattice operations (in particular, the partial order), and convergence in $\mathscr{F}(S)$ are defined pointwise. If $\left\{f_{n}\right\}$ is a sequence in $\mathscr{F}(S)$ converging to an $f \in \mathscr{F}(S)$, we write $\lim f_{n}=f$ or $f_{n} \rightarrow f$; if, in addition, the sequence $\left\{f_{n}\right\}$ is increasing or decreasing, we write $f_{n} \nearrow f$ or $f_{n} \searrow f$, respectively. For $T \subset S$, we shall often identify a function $f \in \mathscr{F}(S)$ and its restriction $f \uparrow T \in \mathscr{F}(T)$.

Throughout this paper, $X$ will be a locally compact Hausdorff space. If $A \subset X$, we denote by $A^{-}$and $A^{\circ}$ the closure and interior of $A$, respectively.

We fix a family $\mathscr{A}$ of subsets of $X$, which satisfies the following conditions.

(i) If $A \in \mathscr{A}$, then $A^{-}$is compact.

(ii) For each $x \in X$, the collection $\mathscr{A}(x)=\left\{A \in \mathscr{A}: x \in A^{\circ}\right\}$ is a neighborhood base at $x$.

(iii) If $A, B \in \mathscr{A}$, then $A \cap B \in \mathscr{A}$, and there are disjoint sets $C_{1}, \ldots, C_{n}$ in $\mathscr{A}$ such that $A-B=\bigcup_{i=1}^{n} C_{i}$.

For $S \subset X$, we let $\mathscr{A}_{S}=\{A \in \mathscr{A}: A \subset S\}$. The next lemma summarizes some basic properties of the family $\mathscr{A}$. For its simple proof, we refer to [5, Section 1]. 
2.1. Lemma. (i) For every collection $\left\{A_{1}, \ldots, A_{m}\right\} \subset \mathscr{A}$ there is a disjoint collection $\left\{B_{1}, \ldots, B_{n}\right\} \subset \mathscr{A}$ such that $\bigcup_{j=1}^{n} B_{j}=\bigcup_{i=1}^{m} A_{i}$, and each $B_{j}$ is contained in some $A_{i}$.

(ii) For every $A \in \mathscr{A}$ and every collection $\left\{A_{1}, \ldots, A_{m}\right\} \subset \mathscr{A}$ there is a disjoint collection $\left\{B_{1}, \ldots, B_{n}\right\} \subset \mathscr{A}_{A}$ with $\bigcup_{j=1}^{n} B_{j}=A-\bigcup_{i=1}^{m} A_{i}$.

(iii) For every $A \in \mathscr{A}$ and every open cover $\mathscr{U}$ of $A^{-}$there is a disjoint collection $\left\{A_{1}, \ldots, A_{m}\right\} \subset \mathscr{A}$ which refines $\mathscr{U}$, and is such that $\cup_{i=1}^{m} A_{i}=A$.

A gauge on $A \in \mathscr{A}$ is a map $U$ which assigns to each $x \in A^{-}$a neighborhood $U(x)$ of $x$ in $X$. The family of all gauges on $A$ is denoted by $\Gamma_{A}$.

A division of $A \in \mathscr{A}$ is a disjoint collection $\left\{A_{1}, \ldots, A_{m}\right\} \subset \mathscr{A}$ with $\bigcup_{i=1}^{m} A_{i}=$ $A$. A partition of $A \in \mathscr{A}$ is a set $P=\left\{\left(A_{1}, x_{1}\right), \ldots,\left(A_{p}, x_{p}\right)\right\}$ such that $\left\{A_{1}, \ldots, A_{p}\right\}$ is a division of $A$ and $\left\{x_{1}, \ldots, x_{p}\right\} \subset A^{-}$. If $U$ is a gauge on $A$, we say that the partition $P$ is $U$-fine whenever $A_{i} \subset U\left(x_{i}\right)$ for $i=1, \ldots, p$. By $\mathscr{P}(A, U)$ we denote the family of all $U$-fine partitions of $A$.

The following lemma is pivotal for our exposition.

\subsection{Lemma. If $A \in \mathscr{A}$ and $U \in \Gamma_{A}$, then $\mathscr{P}(A, U) \neq \varnothing$.}

Proof. By 2.1 (iii), there is a division $\left\{A_{1}, \ldots, A_{p}\right\}$ of $A$ which refines $\{U(x)$ : $\left.x \in A^{-}\right\}$. For each $i=1, \ldots, p$, find an $x_{i} \in A^{-}$with $A_{i} \subset U\left(x_{i}\right)$. Now clearly, $\left\{\left(A_{1}, x_{1}\right), \ldots,\left(A_{p}, x_{p}\right)\right\}$ is a $U$-fine partition of $A$.

Finally, we select a non-negative function $v \in \mathscr{F}(\mathscr{A})$, called volume, such that $v(A)=\sum_{i=1}^{m} v\left(A_{i}\right)$ for each $A \in \mathscr{A}$ and each division $\left\{A_{1}, \ldots, A_{m}\right\}$ of $A$.

Before proceeding further, we give a canonical example of the situation described in this section.

2.3. ExAmple. Let $X=\mathbf{R}, \mathscr{A}=\{[a, b): a, b \in \mathbf{R}, a \leqslant b\}$, and for each $[a, b) \in \mathscr{A}$, let $v([a, b))=\alpha(b)-\alpha(a)$ where $\alpha \in \mathscr{F}(\mathbf{R})$ is an increasing function. We say that the volume $v$ is induced by $\alpha$. If $A \in \mathscr{A}$ and $\delta \in \mathscr{F}\left(A^{-}\right)$is a positive function, we can define a gauge $U_{\delta}$ on $A$ by letting $U_{\delta}(x)=(x-\delta(x), x$ $+\delta(x))$ for each $x \in A^{-}$.

\section{Definition of the integral and its basic properties}

Let $A \in \mathscr{A}$ and $f \in \mathscr{F}\left(A^{-}\right)$. For each partition $P=\left\{\left(A_{1}, x_{1}\right), \ldots,\left(A_{p}, x_{p}\right)\right\}$ of $A$, we set

$$
\sigma(f, P)=\sum_{i=1}^{p} f\left(x_{i}\right) v\left(A_{i}\right)
$$

When no confusion can arise, we write $\sigma(f)$ or $\sigma(P)$ instead of $\sigma(f, P)$. 
3.1 Definition. Let $A \in \mathscr{A}$ and $f \in \mathscr{F}\left(A^{-}\right)$. We say that $f$ is integrable over $A$ if there is an $I \in \mathbf{R}$ with the following property: given $\varepsilon>0$, there is a $U \in \Gamma_{A}$ such that $|\sigma(f, P)-I|<\varepsilon$ for each $P \in \mathscr{P}(A, U)$.

Using Lemma 2.2, it is easy to see that the number $I$ from Definition 3.1 is uniquely determined by $f$. We call it the integral of $f$ over $A$, and denote it by $\int_{A} f$. The family of all functions $f \in \mathscr{F}\left(A^{-}\right)$which are integrable over $A$ is denoted by $\mathscr{R}(A)$.

3.2. REMARK. If we want to emphasize that we are integrating with respect to the volume $v$, we use the symbols $\sigma(f, P ; v), \int_{A} f d v$ and $\mathscr{R}(A ; v)$ the meaning of which is obvious (see Section 4).

The next proposition is a direct consequence of Definition 3.1.

3.3. Proposition. Let $A \in \mathscr{A}$.

(i) $\mathscr{R}(A)$ is a linear subspace of $\mathscr{F}\left(A^{-}\right)$, and the map $f \mapsto \int_{A} f$ is a nonnegative linear functional on $\mathscr{R}(A)$.

(ii) If $c \in \mathbf{R}$ and $f(x)=c$ for each $x \in A^{-}$, then $f \in \mathscr{R}(A)$ and $\int_{A} f=c v(A)$.

(iii) If $f \in \mathscr{R}(A), f \geqslant 0$, and $\int_{A} f=0$, then $g \in \mathscr{R}(A)$ and $\int_{A} g=0$ for each $g \in \mathscr{F}\left(A^{-}\right)$with $|g| \leqslant f$.

The following lemma is a Cauchy test of integrability.

3.4. Lemma. Let $A \in \mathscr{A}$ and $f \in \mathscr{F}\left(A^{-}\right)$. Then $f \in \mathscr{R}(A)$ if and only if for each $\varepsilon>0$ there is a $U \in \Gamma_{A}$ such that $|\sigma(f, P)-\sigma(f, Q)|<\varepsilon$ for every $P, Q \in$ $\mathscr{P}(A, U)$.

Proof. Suppose that the condition of the lemma is satisfied, and choose $U_{n} \in \Gamma_{A}, n=1,2, \ldots$, so that $|\sigma(P)-\sigma(Q)|<1 / n$ for each $P, Q \in \mathscr{P}\left(A, U_{n}\right)$. Replacing $U_{n}(x)$ by $\bigcap_{i=1}^{n} U_{i}(x)$, we may assume that $U_{1}(x) \supset U_{2}(x) \supset \cdots$ for each $x \in A^{-}$. Now if $P_{n} \in \mathscr{P}\left(A, U_{n}\right), n=1,2, \ldots$, then the sequence $\left\{\sigma\left(P_{n}\right)\right\}$ is Cauchy. Let $I=\lim \sigma\left(P_{n}\right)$, and choose an $\varepsilon>0$. There is an integer $n_{0} \geqslant 2 / \varepsilon$ such that $\left|\sigma\left(P_{n_{0}}\right)-I\right|<\varepsilon / 2$. If $P \in \mathscr{P}\left(A, U_{n_{0}}\right)$, then

$$
|\sigma(P)-I| \leqslant\left|\sigma(P)-\sigma\left(P_{n_{0}}\right)\right|+\left|\sigma\left(P_{n_{0}}\right)-I\right|<\frac{1}{n_{0}}+\frac{\varepsilon}{2} \leqslant \varepsilon,
$$

and we see that $\int_{A} f=I$. The converse is obvious.

3.5. Proposition. Let $A \in \mathscr{A}$ and $f \in \mathscr{R}(A)$. Then $f \in \mathscr{R}(B)$ for each $B \in \mathscr{A}_{A}$. 
Proof. Let $\varepsilon>0$. By 3.4, there is a $U \in \Gamma_{A}$ such that $\left|\sigma\left(P^{*}\right)-\sigma\left(Q^{*}\right)\right|<\varepsilon$ for each $P^{*}, Q^{*} \in \mathscr{P}(A, U)$. If $B \in \mathscr{A}_{A}$ then by 2.1 (ii), there are $C_{1}, \ldots, C_{n}$ in $\mathscr{A}$ such that $\left\{B, C_{1}, \ldots, C_{n}\right\}$ is a division of $A$. Use 2.2 to choose $P_{i} \in \mathscr{P}\left(C_{i}, U\right)$, $i=1, \ldots, n$. Now if $P, Q \in \mathscr{P}(B, U)$, then $P^{*}=P \cup\left(\cup_{i=1}^{n} P_{i}\right)$ and $Q^{*}=Q \cup$ $\left(\cup_{i=1}^{n} P_{i}\right)$ belong to $\mathscr{P}(A, U)$. Hence

$$
\varepsilon>\left|\sigma\left(P^{*}\right)-\sigma\left(Q^{*}\right)\right|=|\sigma(P)-\sigma(Q)|,
$$

and the proposition follows from 3.4 .

3.6. Proposition. Let $\left\{A_{1}, \ldots, A_{n}\right\}$ be a division of $A \in \mathscr{A}$, and let $f \in \mathscr{F}\left(A^{-}\right)$. If $f \in \mathscr{R}\left(A_{i}\right), i=1, \ldots, n$, then $f \in \mathscr{R}(A)$ and $\int_{A} f=\sum_{i=1}^{n} \int_{A_{i}} f$.

Proof. Choose $\varepsilon>0$, and find $U_{i} \in \Gamma_{A_{i}}$ so that $\left|\sigma\left(P_{i}\right)-\int_{A_{i}} f\right|<\varepsilon / n$ for each $P_{i} \in \mathscr{P}\left(A_{i}, U_{i}\right), i=1, \ldots, n$. If $x \in A^{-}$, find a neighborhood $V(x)$ of $x$ such that $V(x) \cap A_{i}=\varnothing$ whenever $x \notin A_{i}^{-}$, and let

$$
U(x)=V(x) \cap\left(\cap\left\{U_{i}(x): x \in A_{i}^{-}\right\}\right) .
$$

This defines a gauge $U$ on $A$. Let $P=\left\{\left(B_{1}, x_{1}\right), \ldots,\left(B_{p}, x_{p}\right)\right\}$ be a $U$-fine partition of $A$. By our choice of $U$, we have $A_{i} \cap B_{j}=\varnothing$ whenever $x_{j} \notin A_{i}^{-}$. It follows that

$$
P_{i}=\left\{\left(A_{i} \cap B_{j}, x_{j}\right):\left(B_{j}, x_{j}\right) \in P, x_{j} \in A_{i}^{-}\right\}
$$

is a $U_{i}$-fine partition of $A_{i}, i=1, \ldots, n$, and $\sigma(P)=\sum_{i=1}^{n} \sigma\left(P_{i}\right)$. Consequently

$$
\left|\sigma(P)-\sum_{i=1}^{n} \int_{A_{i}} f\right| \leqslant \sum_{i=1}^{n}\left|\sigma\left(P_{i}\right)-\int_{A_{i}} f\right|<\varepsilon
$$

and the proposition follows.

Next we shall elaborate on Lemma 3.4.

3.7. Lemma. Let $A \in \mathscr{A}$ and $f \in \mathscr{F}\left(A^{-}\right)$. Then $f \in \mathscr{R}(A)$ if and only if for each $\varepsilon>0$ there is a $U \in \Gamma_{A}$ such that $\left|\sigma\left(f, P^{*}\right)-\sigma\left(f, Q^{*}\right)\right|<\varepsilon$ for every $P^{*}=$ $\left\{\left(A_{1}, x_{1}\right), \ldots,\left(A_{n}, x_{n}\right)\right\}$ and $Q^{*}=\left\{\left(A_{1}, y_{1}\right), \ldots,\left(A_{n}, y_{n}\right)\right\}$ from $\mathscr{P}(A, U)$.

Proof. Choose an $\varepsilon>0$, and a $U \in \Gamma_{A}$ so that the condition of the lemma is satisfied. If $P=\left\{\left(B_{1}, x_{1}\right), \ldots,\left(B_{p}, x_{p}\right)\right\}$ and $Q=\left\{\left(C_{1}, y_{1}\right), \ldots,\left(C_{q}, y_{q}\right)\right\}$ belong to $\mathscr{P}(A, U)$, then so do

$$
\begin{aligned}
& P^{*}=\left\{\left(B_{i} \cap C_{j}, x_{i}\right): i=1, \ldots, p ; j=1, \ldots, q\right\}, \\
& Q^{*}=\left\{\left(B_{i} \cap C_{j}, y_{j}\right): i=1, \ldots, p ; j=1, \ldots, q\right\} .
\end{aligned}
$$


Moreover,

$$
\begin{aligned}
\sigma\left(P^{*}\right) & =\sum_{i=1}^{p} \sum_{j=1}^{q} f\left(x_{i}\right) v\left(B_{i} \cap C_{j}\right) \\
& =\sum_{i=1}^{p} f\left(x_{i}\right) \sum_{j=1}^{q} v\left(B_{i} \cap C_{j}\right)=\sum_{i=1}^{p} f\left(x_{i}\right) v\left(B_{i}\right)=\sigma(P),
\end{aligned}
$$

and similarly, $\sigma\left(Q^{*}\right)=\sigma(Q)$. Thus

$$
|\sigma(P)-\sigma(Q)|=\left|\sigma\left(P^{*}\right)-\sigma\left(Q^{*}\right)\right|<\varepsilon,
$$

and $f \in \mathscr{R}(A)$ by 3.4 . The converse is obvious.

3.8. Proposition. Let $A \in \mathscr{A}$ and $f \in \mathscr{F}\left(A^{-}\right)$. Then $f \in \mathscr{R}(A)$ if and only if for each $\varepsilon>0$, there is $U \in \Gamma_{A}$ such that

$$
\sum_{i=1}^{n}\left|f\left(x_{i}\right)-f\left(y_{i}\right)\right| v\left(A_{i}\right)<\varepsilon
$$

for every $P=\left\{\left(A_{1}, x_{1}\right), \ldots,\left(A_{n}, x_{n}\right)\right\}$ and $Q=\left\{\left(A_{1}, y_{1}\right), \ldots,\left(A_{n}, y_{n}\right)\right\}$ from $\mathscr{P}(A, U)$.

Proof. Let $f \in \mathscr{R}(A)$, let $\varepsilon>0$, and choose $U \in \Gamma_{A}$ so that the condition of Lemma 3.7 is satisfied. Let $P=\left\{\left(A_{1}, x_{1}\right), \ldots,\left(A_{n}, x_{n}\right)\right\}$ and $Q=$ $\left\{\left(A_{1}, y_{1}\right), \ldots,\left(A_{n}, y_{n}\right)\right\}$ be in $\mathscr{P}(A, U)$. After a suitable reordering, we may assume that there is an integer $k, 0 \leqslant k \leqslant n$, such that $f\left(x_{i}\right) \geqslant f\left(y_{i}\right)$ for $i=1, \ldots, k$ and $f\left(x_{i}\right)<f\left(y_{i}\right)$ for $i=k+1, \ldots, n$. Clearly

$$
\begin{aligned}
& P^{*}=\left\{\left(A_{1}, x_{1}\right), \ldots,\left(A_{k}, x_{k}\right),\left(A_{k+1}, y_{k+1}\right), \ldots,\left(A_{n}, y_{n}\right)\right\}, \\
& Q^{*}=\left\{\left(A_{1}, y_{1}\right), \ldots,\left(A_{k}, y_{k}\right),\left(A_{k+1}, x_{k+1}\right), \ldots,\left(A_{n}, x_{n}\right)\right\}
\end{aligned}
$$

belong to $\mathscr{P}(A, U)$, and so by our choice of $U$,

$$
\begin{aligned}
\varepsilon & >\left|\sigma\left(P^{*}\right)-\sigma\left(Q^{*}\right)\right| \\
& =\sum_{i=1}^{k}\left[f\left(x_{i}\right)-f\left(y_{i}\right)\right] v\left(A_{i}\right)+\sum_{i=k+1}^{n}\left[f\left(y_{i}\right)-f\left(x_{i}\right)\right] v\left(A_{i}\right) \\
& =\sum_{i=1}^{n}\left|f\left(x_{i}\right)-f\left(y_{i}\right)\right| v\left(A_{i}\right) .
\end{aligned}
$$

The converse follows directly from Lemma 3.7.

3.9. Corollary. Let $A \in \mathscr{A}$ and $f \in \mathscr{R}(A)$. Then $|f| \in \mathscr{R}(A)$ and $\left|\int_{A} f\right| \leqslant$ $\int_{A}|f|$.

Proof. Since ||$f(x)|-| f(y)|| \leqslant|f(x)-f(y)|$, the integrability of $|f|$ follows from 3.8. The inequality is a consequence of 3.3(i). 
3.10. Corollary. If $A \in \mathscr{A}$, then $\mathscr{R}(A)$ is a linear sublattice of $\mathscr{F}\left(A^{-}\right)$.

This corollary follows immediately from 3.9 and 3.3(i).

3.11. Proposition. Let $A \in \mathscr{A}$, and let $f \in \mathscr{F}\left(A^{-}\right)$be continuous. Then $f \in \mathscr{R}(A)$.

Proof. Choose an $\varepsilon>0$, and find a $U \in \Gamma_{A}$ so that, given $x \in A^{-}$, we have $|f(x)-f(y)|<\varepsilon$ for each $y \in A^{-} \cap U(x)$. Let $P=\left\{\left(A_{1}, x_{1}\right), \ldots,\left(A_{n}, x_{n}\right)\right\}$ and $Q=\left\{\left(A_{1}, y_{1}\right), \ldots,\left(A_{n}, y_{n}\right)\right\}$ belong to $\mathscr{P}(A, U)$. With no loss of generality, we may assume that all the $A_{i}$ 's are nonempty; for the volume is finite and additive, and hence $v(\varnothing)=0$. Now for $i=1, \ldots, n$, choose $z_{i} \in A_{i}$. Since $A_{i} \subset U\left(x_{i}\right) \cap$ $U\left(y_{i}\right)$, we obtain

$$
\begin{aligned}
\sum_{i=1}^{n}\left|f\left(x_{i}\right)-f\left(y_{i}\right)\right| v\left(A_{i}\right) & \leqslant \sum_{i=1}^{n}\left[\left|f\left(x_{i}\right)-f\left(z_{i}\right)\right|+\left|f\left(z_{i}\right)-f\left(y_{i}\right)\right|\right] v\left(A_{i}\right) \\
& \leqslant 2 \varepsilon \sum_{i=1}^{n} v\left(A_{i}\right)=2 \varepsilon v(A) .
\end{aligned}
$$

The proposition follows from 3.8 .

\section{Change of volume}

Let $A \in \mathscr{A}$. A subpartition of $A$ is a set $S=\left\{\left(A_{1}, x_{1}\right), \ldots,\left(A_{s}, x_{s}\right)\right\}$ such that $A_{1}, \ldots, A_{s}$ are disjoint sets from $\mathscr{A}_{A}$ and $x_{1}, \ldots, x_{s}$ are points from $A^{-}$. Thus for a subpartition $S$ we do not require that $\bigcup_{i=1}^{s} A_{i}=A$. If $U \in \Gamma_{A}$, we say that the subpartition $S$ is $U$-fine whenever $A_{i} \subset U\left(x_{i}\right)$ for $i=1, \ldots, s$. Given a function $f \in \mathscr{F}\left(A^{-}\right)$, we let

$$
\boldsymbol{\sigma}(f, S)=\sum_{i=1}^{s} f\left(x_{i}\right) v\left(A_{i}\right)
$$

4.1. Lemma. Let $A \in \mathscr{A}$ and $U \in \Gamma_{A}$. Each $U$-fine subpartition of $A$ is a subset of a $U$-fine partition of $A$.

Proof. If $S=\left\{\left(A_{1}, x_{1}\right), \ldots,\left(A_{s}, x_{s}\right)\right\}$ is a $U$-fine subpartition of $A$, then by 2.1(ii), we can find sets $B_{1}, \ldots, B_{n}$ in $\mathscr{A}_{A}$ so that $\left\{A_{1}, \ldots, A_{s}, B_{1}, \ldots, B_{n}\right\}$ is a division of $A$. By Lemma 2.2, there are $Q_{i} \in \mathscr{P}\left(B_{i}, U\right), i=1, \ldots, n$, and so $P=S \cup\left(\cup_{i-1}^{n} Q_{i}\right)$ is a $U$-fine partition of $A$.

Next we shall prove an important proposition which is sometimes referred to as the Henstock lemma. 
4.2. Proposition. Let $A \in \mathscr{A}$ and $f \in \mathscr{R}(A)$. For each $\varepsilon>0$ there is a $U \in \Gamma_{A}$ such that

$$
\sum_{i=1}^{s}\left|f\left(x_{i}\right) v\left(A_{i}\right)-\int_{A_{i}} f\right|<\varepsilon
$$

for every $U$-fine subpartition $\left\{\left(A_{1}, x_{1}\right), \ldots,\left(A_{s}, x_{s}\right)\right\}$ of $A$.

Proof. Let $\varepsilon>0$. By the definition of integrability, there is a $U \in \Gamma_{A}$ such that $\left|\sigma(P)-\int_{A} f\right|<\varepsilon / 3$, for each $P \in \mathscr{P}(A, U)$. In view of 4.1 , it suffices to consider only $U$-fine partitions of $A$. So choose $\left\{\left(A_{1}, x_{1}\right), \ldots,\left(A_{n}, x_{n}\right)\right\}$ in $\mathscr{P}(A, U)$. After a suitable reordering, we may assume that there is an integer $k, 0 \leqslant k \leqslant n$, such that $f\left(x_{i}\right) v\left(A_{i}\right)-\int_{A_{i}} f$ is nonnegative for $i=1, \ldots, k$ and negative for $i=k+$ $1, \ldots, n$. Using 3.5 , we can find $P_{i} \in \mathscr{P}\left(A_{i}, U\right)$ so that $\left|\sigma\left(P_{i}\right)-\int_{A_{i}} f\right|<\varepsilon / 3 n$, $i=1, \ldots, n$. Now

$$
\begin{gathered}
P=\left\{\left(A_{1}, x_{1}\right), \ldots,\left(A_{i}, x_{i}\right)\right\} \cup P_{k+1} \cup \cdots \cup P_{n}, \\
Q=\left\{\left(A_{k+1}, x_{k+1}\right), \ldots,\left(A_{n}, x_{n}\right)\right\} \cup P_{1} \cup \cdots \cup P_{k}
\end{gathered}
$$

belong to $\mathscr{P}(A, U)$. Thus

$$
\begin{aligned}
\frac{\varepsilon}{3} & >\left|\sigma(P)-\int_{A} f\right| \geqslant \sum_{i=1}^{k}\left[f\left(x_{i}\right) v\left(A_{i}\right)-\int_{A_{i}} f\right]-\left|\sum_{i=k+1}^{n}\left[\sigma\left(P_{i}\right)-\int_{A_{i}} f\right]\right| \\
& \geqslant \sum_{i=1}^{k}\left|f\left(x_{i}\right) v\left(A_{i}\right)-\int_{A_{i}} f\right|-(n-k) \frac{\varepsilon}{3 n},
\end{aligned}
$$

and similarly,

$$
\frac{\varepsilon}{3}>\sum_{i=k+1}^{n}\left|f\left(x_{i}\right) v\left(A_{i}\right)-\int_{A_{i}} f\right|-k \frac{\varepsilon}{3 n} .
$$

From this we obtain $\sum_{i=1}^{n}\left|f\left(x_{i}\right) v\left(A_{i}\right)-\int_{A_{i}} f\right|<\varepsilon$, and the proposition is proved.

Let $g \in \mathscr{F}(X)$ be a nonnegative function such that $g \in \mathscr{R}(A ; v)$ for each $A \in \mathscr{A}$ (see Remark 3.2). In view of 3.3(i) and 3.6, we can define a volume $v_{g}$ by setting $v_{g}(A)=\int_{A} g d v$ for each $A \in \mathscr{A}$. The following proposition relates the integrals with respect to volumes $v$ and $v_{g}$.

4.3. Proposition. Let $A \in \mathscr{A}$ and $f \in \mathscr{F}\left(A^{-}\right)$. Then $f \in \mathscr{R}\left(A ; v_{g}\right)$ if and only if $f g \in \mathscr{R}(A ; v)$, in which case $\int_{A} f d v_{g}=\int_{A} f g d v$.

Proof. Choose $\varepsilon>0$, and use 4.2 to find $U_{n} \in \Gamma_{A}, n=1,2, \ldots$, so that

$$
\sum_{i=1}^{s}\left|g\left(x_{i}\right) v\left(A_{i}\right)-v_{g}\left(A_{i}\right)\right|<\frac{\varepsilon}{n 2^{n}}
$$


for each $U_{n}$-fine subpartition $\left\{\left(A_{1}, x_{1}\right), \ldots,\left(A_{s}, x_{s}\right)\right\}$ of $A$. If $E_{n}=\left\{x \in A^{-}\right.$: $n-1 \leqslant|f(x)|<n\}, n=1,2, \ldots$, then $A^{-}$is a disjoint union of the $E_{n}$ 's. Thus we can define a $U \in \Gamma_{A}$ by setting $U(x)=U_{n}(x)$ whenever $x \in E_{n}$. Let $Q=$ $\left\{\left(B_{1}, y_{1}\right), \ldots,\left(B_{q}, y_{q}\right)\right\}$ be in $\mathscr{P}(A, U)$. For $n=1,2, \ldots$, the set $\left\{\left(B_{i}, y_{i}\right)\right.$ : $\left.y_{i} \in E_{n}\right\}$ is a $U_{n}$-fine subpartition of $A$. Thus

$$
\begin{aligned}
\mid \sigma(f g, P ; v)-\sigma & \left(f, P ; v_{g}\right)\left|\leqslant \sum_{i=1}^{q}\right| f\left(y_{i}\right)|\cdot| g\left(y_{i}\right) v\left(B_{i}\right)-v_{g}\left(B_{i}\right) \mid \\
& =\sum_{n=1}^{\infty} \sum_{y_{i} \in E_{n}}\left|f\left(y_{i}\right)\right| \cdot\left|g\left(y_{i}\right) v\left(B_{i}\right)-v_{g}\left(B_{i}\right)\right|<\sum_{n=1}^{\infty} n \frac{\varepsilon}{n 2^{n}}=\varepsilon,
\end{aligned}
$$

and the proposition easily follows.

\section{The convergence theorems}

We begin with the fundamental monotone convergence theorem.

5.1. TheOREM. Let $A \in \mathscr{A}, f \in \mathscr{F}\left(A^{-}\right)$, and let $f_{n} \in \mathscr{R}(A), n=1,2, \ldots$ If $f_{n}>f$ and $\lim \int_{A} f_{n}<+\infty$, then $f \in \mathscr{R}(A)$ and $\int_{A} f=\lim \int_{A} f_{n}$.

Proof. Choose an $\varepsilon>0$, and using Proposition 4.2, find $U_{n} \in \Gamma_{A}, n=1$, $2, \ldots$, so that $\sum_{i=1}^{s}\left|f_{n}\left(y_{i}\right) v\left(B_{i}\right)-\int_{B_{i}} f_{n}\right|<\varepsilon 2^{-n}$ for each $U_{n}$-fine subpartition $\left\{\left(B_{1}, y_{1}\right), \ldots,\left(B_{s}, y_{s}\right)\right\}$ of $A$. Let $I=\lim \int_{A} f_{n}$, and select an integer $n_{0} \geqslant 1$ with $\int_{A} f_{n_{0}}>I-\varepsilon$. Finally, for each $x \in A^{-}$, find an integer $n(x) \geqslant n_{0}$ such that $\left|f_{n(x)}(x)-f(x)\right|<\varepsilon$. Now define a $U \in \Gamma_{A}$ by setting $U(x)=U_{n(x)}(x)$ for every $x \in A^{-}$, and let $P=\left\{\left(A_{1}, x_{1}\right), \ldots,\left(A_{p}, x_{p}\right)\right\}$ be in $\mathscr{P}(A, U)$. We shall prove the theorem by showing that $|\sigma(f, P)-I|<\varepsilon[2+v(A)]$.

To this end, we first observe that

$$
\begin{aligned}
\left|\sigma(f, P)-\sum_{i=1}^{p} f_{n\left(x_{i}\right)}\left(x_{i}\right) v\left(A_{i}\right)\right| & \leqslant \sum_{i=1}^{p}\left|f\left(x_{i}\right)-f_{n\left(x_{i}\right)}\left(x_{i}\right)\right| v\left(A_{i}\right) \\
& \leqslant \varepsilon \sum_{i=1}^{p} v\left(A_{i}\right)=\varepsilon v(A) .
\end{aligned}
$$

The integers $n\left(x_{i}\right)$ need not be distinct. Let

$$
\left\{n\left(x_{1}\right), \ldots, n\left(x_{p}\right)\right\}=\left\{k_{1}, \ldots, k_{q}\right\}
$$


where $k_{1}<\cdots<k_{q}$. Then $\{1, \ldots, p\}$ is the disjoint union of the sets $T_{j}=\{i$ : $\left.n\left(x_{i}\right)=k_{j}\right\}, j=1, \ldots, q$. Moreover, the set $\left\{\left(A_{i}, x_{i}\right): i \in T_{j}\right\}$ is a $U_{k_{j}}$-fine subpartition of $A$; for if $i \in T_{j}$, then

$$
A_{i} \subset U\left(x_{i}\right)=U_{n\left(x_{i}\right)}\left(x_{i}\right)=U_{k_{j}}\left(x_{i}\right) .
$$

Therefore

$$
\begin{aligned}
(* *)\left|\sum_{i=1}^{p} f_{n\left(x_{i}\right)}\left(x_{i}\right) v\left(A_{i}\right)-\sum_{i=1}^{p} \int_{A_{i}} f_{n\left(x_{i}\right)}\right| & \leqslant \sum_{j=1}^{q} \sum_{i \in T_{j}}\left|f_{k_{j}}\left(x_{i}\right) v\left(A_{i}\right)-\int_{A_{i}} f_{k_{j}}\right| \\
& <\sum_{j=1}^{q} \varepsilon 2^{-k j}<\varepsilon \sum_{k=1}^{\infty} 2^{-k}=\varepsilon .
\end{aligned}
$$

Since $n_{0} \leqslant n\left(x_{i}\right) \leqslant k_{q}$ for $i=1, \ldots, p$, using 3.5 and 3.6, we obtain

$$
\begin{aligned}
I-\varepsilon & <\int_{A} f_{n_{0}}=\sum_{i=1}^{p} \int_{A_{i}} f_{n_{0}} \leqslant \sum_{i=1}^{p} \int_{A_{i}} f_{n\left(x_{i}\right)} \leqslant \sum_{i=1}^{p} \int_{A_{i}} f_{k_{q}} \\
& =\int_{A} f_{k_{q}} \leqslant I<I+\varepsilon,
\end{aligned}
$$

and consequently $\left|\sum_{i=1}^{p} \int_{A_{i}} f_{n\left(x_{i}\right)}-I\right|<\varepsilon$. This in conjunction with (*) and (**) implies the desired inequality.

5.2. Corollary. Let $A \in \mathscr{A}, f_{n}, g \in \mathscr{R}(A)$, and $\left|f_{n}\right| \leqslant g$ for $n=1,2, \ldots$ Then $\sup f_{n}$ and $\inf f_{n}$ belong to $\mathscr{R}(A)$.

By a standard argument, this corollary follows directly from 3.10 and 5.1.

Omitting the familiar proofs, we list the Fatou lemma and the dominated convergence theorem.

5.3. Proposition. Let $A \in \mathscr{A}, f_{n}, g \in \mathscr{R}(A)$, and $\left|f_{n}\right| \leqslant g$ for $n=1,2, \ldots$ Then $\lim \sup f_{n}$ and $\liminf f_{n}$ belong to $\mathscr{R}(A)$ and

$$
\int_{A} \liminf f_{n} \leqslant \liminf \int_{A} f_{n} \leqslant \limsup \int_{A} f_{n} \leqslant \int_{A} \limsup f_{n} .
$$

5.4. Corollary. Let $A \in \mathscr{A}, f_{n}, g \in \mathscr{R}(A)$, and $\left|f_{n}\right| \leqslant g$ for $n=1,2, \ldots$. If $f_{n} \rightarrow f$, then $f \in \mathscr{R}(A)$ and $\int_{A} f=\lim \int_{A} f_{n}$. 
From this, and from 3.11 and 3.3(ii), we obtain the following corollary.

5.5. Corollary. Let $A \in \mathscr{A}$. Then each bounded Baire function on $A^{-}$is integrable over $A$.

For the definition of the Baire functions we refer to [8, (3-13), page 41].

Let $T$ be a set. A family $\mathscr{E} \subset \mathscr{F}(T)$ is called directed upward or downward if $\mathscr{E} \neq \varnothing$ and for each $f, g \in \mathscr{E}$ there is an $h \in \mathscr{E}$ such that $f \vee g \leqslant h$ or $h \leqslant f \wedge g$, respectively.

5.6. THEOREM. Let $A \in \mathscr{A}, g \in \mathscr{F}\left(A^{-}\right)$, and let $\mathscr{E} \subset \mathscr{F}\left(A^{-}\right)$be an upward directed family of continuous functions. If $g=\sup \mathscr{E}$ and $\sup \left\{\int_{A} f: f \in \mathscr{E}\right\}<+\infty$, then $g \in \mathscr{R}(A)$ and $\int_{A} g=\sup \left\{\int_{A} f: f \in \mathscr{E}\right\}$.

Proof. Choose an $\varepsilon>0$, and for each $f \in \mathscr{E}$ find an $U_{f} \in \Gamma_{A}$ so that, given $x \in A^{-}$, we have $|f(x)-f(y)|<\varepsilon$ for every $y \in A^{-} \cap U_{f}(x)$. It follows from 3.3(i), (ii) that $\left|f(x) v(B)-\int_{B} f\right| \leqslant \varepsilon v(B)$ for each $B \in \mathscr{A}_{U_{f}(x)}$. Let $I=\sup \left\{\int_{A} f\right.$ : $f \in \mathscr{E}\}$, and select an $h \in \mathscr{E}$ with $\int_{A} h>I-\varepsilon$. Finally, for each $x \in A^{-}$, find an $f_{x} \in \mathscr{E}$ such that $f_{x} \geqslant h$ and $f_{x}(x)>g(x)-\varepsilon$. Now define a $U \in \Gamma_{A}$ by setting $U(x)=U_{f_{x}}(x)$ for every $x \in A^{-}$, and let $P=\left\{\left(A_{1}, x_{1}\right), \ldots,\left(A_{p}, x_{p}\right)\right\}$ be in $\mathscr{P}(A, U)$. We shall prove the theorem by showing that $|\sigma(g, P)-I|<$ $\varepsilon[1+2 v(A)]$.

First we have

$$
\begin{aligned}
\left|\sigma(g, P)-\sum_{i=1}^{p} \int_{A_{i}} f_{x_{i}}\right| \leqslant & \left|\sum_{i=1}^{p} g\left(x_{i}\right) v\left(A_{i}\right)-\sum_{i=1}^{p} f_{x_{i}}\left(x_{i}\right) v\left(A_{i}\right)\right| \\
& +\left|\sum_{i=1}^{p} f_{x_{i}}\left(x_{i}\right) v\left(A_{i}\right)-\sum_{i=1}^{p} \int_{A_{i}} f_{x_{i}}\right| \\
& \leqslant \sum_{i=1}^{p}\left\{\left[g\left(x_{i}\right)-f_{x_{i}}\left(x_{i}\right)\right] v\left(A_{i}\right)+\left|f_{x_{i}}\left(x_{i}\right) v\left(A_{i}\right)-\int_{A_{i}} f_{x_{i}}\right|\right\} \\
& \leqslant \sum_{i=1}^{p} 2 \varepsilon v\left(A_{i}\right)=2 \varepsilon v(A) ;
\end{aligned}
$$

for $A_{i} \subset U\left(x_{i}\right)=U_{f_{x_{i}}}\left(x_{i}\right), i=1, \ldots, p$. There is a $\psi \in \mathscr{E}$ with $\psi \geqslant \bigvee_{i=1}^{p} f_{x_{i}}$. By 3.5 and 3.6,

$$
I-\varepsilon<\int_{A} h=\sum_{i=1}^{p} \int_{A_{i}} h \leqslant \sum_{i=1}^{p} \int_{A_{i}} f_{x_{i}} \leqslant \sum_{i=1}^{p} \int_{A_{i}} \psi=\int_{A} \psi \leqslant I<I+\varepsilon,
$$

and consequently $\left|\sum_{i=1}^{p} \int_{A_{i}} f_{x_{i}}-I\right|<\varepsilon$. The desired inequality follows. 
5.7. Corollary. Let $A \in \mathscr{A}$. Then each bounded lower or upper semicontinuous function on $A^{-}$is integrable over $A$.

This corollary follows from Theorem 5.6 and [8, (3.5), page 32].

\section{The relationship to the Lebesgue integral}

We begin with the definition of a lower derivative of a set function. For this purpose it will be convenient to introduce the extended real numbers $\mathbf{R} U$ $\{-\infty,+\infty\}$, and for $a \in \mathbf{R}$ let $a / 0=+\infty$ if $a \geqslant 0$, and $a / 0=-\infty$ if $a<0$.

Let $A \in \mathscr{A}$ and $M \in \mathscr{F}\left(\mathscr{A}_{A}\right)$. For $x \in A^{-}$, we set

$$
{ }_{*} M(x)=\sup _{V(x)} \inf \left\{\frac{M(B)}{v(B)}: B \in \mathscr{A}_{A \cap V(x)}\right\}
$$

where the supremum is taken over all neighborhoods $V(x)$ of $x$. The map $x \rightarrow{ }_{*} M(x)$ is an extended real-valued function on $A^{-}$, denote it by ${ }_{*} M$.

\subsection{Lemma. Let $A \in \mathscr{A}$ and $M \in \mathscr{F}\left(\mathscr{A}_{A}\right)$. Then ${ }_{*} M$ is lower semicontinuous.}

Proof. Let $x \in A^{-}$, and let ${ }_{*} M(x)>c$ for some $c \in \mathbf{R}$. Then there is an open neighborhood $V(x)$ of $x$ such that

$$
c<c^{\prime}=\inf \left\{\frac{M(B)}{v(B)}: B \in \mathscr{A}_{A \cap V(x)}\right\},
$$

and it follows that $* M(y) \geqslant c^{\prime}>c$ for each $y \in V(x)$.

6.2. LemMa. Let $A \in \mathscr{A}$ and $f \in \mathscr{R}(A)$ be bounded from above. Then for each $\varepsilon>0$ there is a bounded lower semicontinuous function $g \in \mathscr{R}(A)$ such that $f \leqslant g$ and $\int_{A} g<\int_{A} f+\varepsilon$.

Proof. Choose an $\varepsilon>0$ and using 4.2, find a $U \in \Gamma_{A}$ so that $\sum_{i=1}^{s} \mid f\left(y_{i}\right) v\left(B_{i}\right)$ $-\int_{B_{i}} f \mid<\varepsilon / 3$ for each $U$-fine subpartition $\left\{\left(B_{1}, y_{1}\right), \ldots,\left(B_{s}, y_{s}\right)\right\}$ of $A$. For $B \in \mathscr{A}_{A}$, let $M(B)=\sup _{S} \sigma(f, S)$, where the supremum is taken over all $U$-fine subpartitions $S=\left\{\left(B_{1}, y_{1}\right), \ldots,\left(B_{s}, y_{s}\right)\right\}$ of $A$ for which $\cup_{i=1}^{s} B_{i}=B$. If $S$ is such a subpartition of $A$, then $\left|\sigma(f, S)-\int_{B} f\right| \leqslant \sum_{i=1}^{s}\left|f\left(y_{i}\right) v\left(B_{i}\right)-\int_{B_{i}} f\right|<\varepsilon / 3$; and it follows that $\left|M(B)-\int_{B} f\right| \leqslant \varepsilon / 3$. In particular, the map $B \mapsto M(B)$ belongs to $\mathscr{F}\left(\mathscr{A}_{A}\right)$.

For $x \in A^{-}$, we have ${ }_{*} M(x) \geqslant f(x)$. Indeed if $B \in \mathscr{A}_{A \cap U(x)}$, then $\{(B, x)\}$ is a $U$-fine subpartition of $A$, and hence $M(B) \geqslant f(x) v(B)$. Thus if $c \in \mathbf{R}$ is an upper bound of $f$, then $g={ }_{*} M \wedge c$ is a lower semicontinuous function on $A^{-}$, 
and $g \geqslant f$. Since $A^{-}$is compact, $g$ is bounded, and by 5.7, it belongs to $\mathscr{R}(A)$. Hence there is a $V \in \Gamma_{A}$ such that $\left|\sigma(g, P)-\int_{A} g\right|<\varepsilon / 3$ for each $P \in \mathscr{P}(A, V)$. Let $\alpha=\varepsilon / 3[v(A)+1]$ and $x \in A^{-}$. As ${ }_{*} M(x)>g(x)-\alpha$, there is a neighborhood $W(x)$ of $x$ such that $W(x) \subset V(x)$ and $M(B) \geqslant[g(x)-\alpha] v(B)$ for each $B \in \mathscr{A}_{A \cap W(x)}$. The map $x \mapsto W(x)$ defines a gauge $W$ on $A$. If $P=$ $\left\{\left(A_{1}, x_{1}\right), \ldots,\left(A_{p}, x_{p}\right)\right\}$ belongs to $\mathscr{P}(A, W)$, it belongs also to $\mathscr{P}(A, V)$, and we have

$$
\begin{aligned}
\int_{A} g & <\sigma(g, P)+\frac{\varepsilon}{3}=\sum_{i=1}^{p} g\left(x_{i}\right) v\left(A_{i}\right)+\frac{\varepsilon}{3} \leqslant \sum_{i=1}^{p}\left[M\left(A_{i}\right)+\alpha v\left(A_{i}\right)\right]+\frac{\varepsilon}{3} \\
& =\sum_{i=1}^{p} M\left(A_{i}\right)+\alpha v(A)+\frac{\varepsilon}{3}<\sum_{i=1}^{p} M\left(A_{i}\right)+\frac{2 \varepsilon}{3}
\end{aligned}
$$

It remains to estimate $\sum_{i=1}^{p} M\left(A_{i}\right)$. To this end, for $i=1, \ldots, p$, choose a $U$-fine subpartition $S_{i}=\left\{\left(C_{1}^{i}, z_{1}^{i}\right), \ldots,\left(C_{s_{i}}^{i}, z_{s_{i}}^{i}\right)\right\}$ of $A$ with $\bigcup_{j=1}^{s_{i}} C_{j}^{i}=A_{i}$. Then $S=\bigcup_{i=1}^{p} S_{i}$ belongs to $\mathscr{P}(A, U)$, and we have

$$
\sum_{i=1}^{p} \sigma\left(f, S_{i}\right)=\sigma(f, S) \leqslant M(A) \leqslant \int_{A} f+\frac{\varepsilon}{3} .
$$

From the arbitrariness of the $S_{i}$ 's, we obtain $\sum_{i=1}^{p} M\left(A_{i}\right) \leqslant \int_{A} f+\varepsilon / 3$, and the lemma follows.

If ( $X, \mathscr{M}, \mu$ ) is a measure space, and if $E \in \mathscr{M}$, we denote by $\mathscr{L}_{\mu}(E)$ the family of all functions $f \in \mathscr{F}\left(E^{-}\right)$for which the finite Lebesgue integral $(L) \int_{E} f d \mu$ exists.

Throughout the remainder of this section, we shall assume that there is a measure space $(X, \mathscr{M}, \mu)$ such that $\mathscr{A} \subset \mathscr{M}$ and $\mu(A)=v(A)$ for each $A \in \mathscr{A}$. Since $v$ is finite and $\mathscr{A}$ contains a neighborhood of each $x \in X$, the measure $\mu$ is locally finite, i.e., $\mu(E)<+\infty$ for each $E \in \mathscr{M}$ with $E^{-}$compact.

6.3. Proposition. Let $A \in \mathscr{A}$, and let $f \in \mathscr{L}_{\mu}(A)$ be continuous. Then $\int_{A} f=$ $(L) \int_{A} f d \mu$.

Proof. Choose an $\varepsilon>0$, and find a $U \in \Gamma_{A}$ so that, given $x \in A^{-}$, we have $|f(x)-f(y)|<\varepsilon$ for each $y \in A^{-} \cap U(x)$. It follows that

$$
\left|f(x) \mu(E)-(L) \int_{E} f d \mu\right|<\varepsilon \mu(E)
$$


for each $E \in \mathscr{M}$ with $E \subset A \cap U(x)$. Now if $P=\left\{\left(A_{1}, x_{1}\right), \ldots,\left(A_{p}, x_{p}\right)\right\}$ is in $\mathscr{P}(A, U)$, we have

$$
\begin{aligned}
\left|\sigma(f, P)-(L) \int_{A} f d \mu\right| & \leqslant \sum_{i=1}^{p}\left|f\left(x_{i}\right) v\left(A_{i}\right)-(L) \int_{A_{i}} f d \mu\right| \\
& \leqslant \sum_{i=1}^{p} \varepsilon v\left(A_{i}\right)=\varepsilon v(A),
\end{aligned}
$$

and the proposition follows.

Applying 5.5, we obtain the following corollary.

6.4. Corollary. Let $A \in \mathscr{A}$, and let $f \in \mathscr{L}_{\mu}(A)$ be a bounded Baire function. Then $\int_{A} f=(L) \int_{A} f d \mu$.

The next example shows that, in general, Corollary 6.4 cannot be extended to bounded Borel functions in $\mathscr{L}_{\mu}(A)$, i.e., those bounded functions in $\mathscr{L}_{\mu}(A)$ which are measurable with respect to the Borel $\sigma$-algebra in $A^{-}$(see $[8,(7.4)$, page 82]).

6.5. EXAMPLE. Let $X$ be the compact Hausdorff space of all ordinals less than or equal to $\omega_{1}$ (the first uncountable ordinal), let $\mathscr{A}=\mathscr{M}$ be the Borel $\sigma$-algebra in $X$, and let $v=\mu$ be the Dieudonné measure on $\mathscr{K}$ (see [8, (9-10), page 116]). Let $A=X-\left\{\omega_{1}\right\}$, and on $A^{-}=X$ define a bounded Borel function $f$ by setting $f\left(\omega_{1}\right)=1$ and $f(x)=0$ for each $x \in A$. Then $f \in \mathscr{L}_{\mu}(A)$ and $(L) \int_{A} f d \mu$ $=0$. However, choosing a $U \in \Gamma_{A}$ so that $U(x)$ is countable for each $x \in A$, it is easy to see that $f \in \mathscr{R}(A)$ and $\int_{A} f=1$.

To avoid the pathology of the previous example, we shall assume throughout that the measure space $(X, \mathscr{M}, \mu)$ is locally Radon, i.e., that $\mathscr{M}$ contains all compact subsets of $X$, and that

$$
\mu(E)=\sup \{\mu(K): K \text { compact, } K \subset E\}
$$

for each $E \in \mathscr{M}$ with $E^{-}$compact. Since $\mu$ is locally finite, we also have

$$
\mu(E)=\inf \{\mu(G): G \in \mathscr{M}, G \text { open, } E \subset G\}
$$

for each $E \in \mathscr{M}$ with $E^{-}$compact.

The characteristic function of a set $E \subset X$ is the function $\chi_{E} \in \mathscr{F}(X)$ defined by $\chi_{E}(x)=1$ if $x \in E$, and $\chi_{E}(x)=0$ if $x \in X-E$.

A set $E \subset X$ is called negligible if there is an $H \in \mathscr{M}$ such that $E \subset H$ and $\mu(H)=0$.

6.6. Lemma. Let $A \in \mathscr{A}$, and let $E \subset X$ be negligible. Then $\chi_{E} \in \mathscr{R}(A)$ and $\int_{A} \chi_{E}=0$. 
Proof. Given $\varepsilon>0$, there is an open set $G \in \mathscr{M}$ for which $E \cap A^{-} \subset G$ and $\mu(G)<\varepsilon$. Let $U \in \Gamma_{A}$ be such that $U(x) \subset G$ for each $x \in E \cap A^{-}$, and let $P=\left\{\left(A_{1}, x_{1}\right), \ldots,\left(A_{p}, x_{p}\right)\right\}$ be in $\mathscr{P}(A, U)$. Then $0 \leqslant \sigma\left(\chi_{E}, P\right)=\Sigma_{x_{i} \in E} v\left(A_{i}\right)$ $\leqslant \mu(G)<\varepsilon$, and the lemma is proved.

6.7. Proposition. Let $A \in \mathscr{A}, f, g \in \mathscr{F}\left(A^{-}\right)$, and let $\left\{x \in A^{-}: f(x) \neq g(x)\right\}$ be a negligible set. Then $f \in \mathscr{R}(A)$ if and only if $g \in \mathscr{R}(A)$, in which case $\int_{A} f=\int_{A} g$.

Proof. If $h=f-g$, then the set $E=\left\{x \in A^{-}: h(x) \neq 0\right\}$ is negligible. By 6.6 and 3.3(i), (iii), the functions $h_{n}=|h| \wedge\left(n \chi_{E}\right)$ belong to $\mathscr{R}(A)$, and $\int_{A} h_{n}=0$, $n=1,2, \ldots$ As $h_{n} \rtimes|h|$, Theorem 5.1 implies that $|h| \in \mathscr{R}(A)$ and $\int_{A}|h|=0$. The proposition follows by 3.3 (iii).

6.8. Lemma. Let $A \in \mathscr{A}$ and $E \in \mathscr{M}$. Then $\chi_{E} \in \mathscr{R}(A)$ and $\int_{A} \chi_{E}=\mu(A \cap E)$.

Proof. Assume first that $E$ is compact, and find open sets $G_{n} \in \mathscr{M}$ such that $E \subset G_{n+1} \subset G_{n}, n=1,2, \ldots$, and $\mu\left(\cap_{n=1}^{\infty} G_{n}-E\right)=0$. There are continuous functions $f_{n} \in \mathscr{F}(X)$ with $\chi_{E} \leqslant f_{n} \leqslant \chi_{G_{n}}$. It follows that $f_{n} \rightarrow \chi_{E}$ almost everywhere, and so by $5.4,6.7$, and 6.3 , we obtain $\chi_{E} \in \mathscr{R}(A)$ and

$$
\int_{A} \chi_{E}=\lim \int_{A} f_{n}=\lim (L) \int_{A} f_{n} d \mu=(L) \int_{A} \chi_{E} d \mu .
$$

For an arbitrary $E \in \mathscr{M}$ there is an increasing sequence $\left\{K_{n}\right\}$ of compact sets contained in $E \cap A^{-}$, and such that $\mu\left(E \cap A^{-}-\bigcup_{n=1}^{\infty} K_{n}\right)=0$. Thus $\chi_{K_{n}} \rightarrow$ $\chi_{E \cap A^{-}}$almost everywhere, and so by $5.4,6.7$, and the first part of our proof, we have $\chi_{E} \in \mathscr{R}(A)$ and

$$
\begin{aligned}
\int_{A} \chi_{E} & =\int_{A} \chi_{E \cap A^{-}}=\lim \int_{A} \chi_{K_{n}}=\lim (L) \int_{A} \chi_{K_{n}} d \mu \\
& =(L) \int_{A} \chi_{E \cap A^{-}} d \mu=\mu(A \cap E) .
\end{aligned}
$$

6.9. TheOREM. Let $A \in \mathscr{A}$. Then $\mathscr{L}_{\mu}(A) \subset \mathscr{R}(A)$ and $\int_{A} f=(L) \int_{A} f d \mu$ for each $f \in \mathscr{L}_{\mu}(A)$.

Proof. It follows from 3.3(i) and 6.8 that each $\mathscr{M}$-simple function $f$ (see [8, (7.25), page 88]) belongs to $\mathscr{R}(A)$, and that $\int_{A} f=(L) \int_{A} f d \mu$. In view of $[8,(7.29)$, page 90] and 5.1, this extends to each nonnegative function $f \in \mathscr{L}_{\mu}(A)$, and the theorem follows. 
Clearly, for an $A \in \mathscr{A}$ the set $A^{-}-A$ need not be negligible. However, we still have the following corollary.

6.10. Corollary. Let $A \in \mathscr{A}$, and let $f, g \in \mathscr{F}\left(A^{-}\right)$be such that $f(x)=g(x)$ for each $x \in A$. Then $f \in \mathscr{R}(A)$ if and only if $g \in \mathscr{R}(A)$, in which case $\int_{A} f=\int_{A} g$.

Since $f-g$ belongs to $\mathscr{L}_{\mu}(A)$, and since $(L) \int_{A}(f-g) d \mu=0$, this corollary follows immediately from Theorem 6.9 .

6.11. REMARK. Example 6.5 shows that statements 6.6 through 6.10 are false if the measure space $(X, \mathscr{M}, \mu)$ is not locally Radon.

6.12. TheOREM. If the measure $\mu$ is complete, then $\mathscr{R}(A)=\mathscr{L}_{\mu}(A)$ for each $A \in \mathscr{A}$.

Proof. Let $A \in \mathscr{A}$, and let $f \in \mathscr{R}(A)$ be bounded. By 6.2, there are bounded lower semicontinuous functions $g_{n} \in \mathscr{R}(A)$ and bounded upper semicontinuous functions $h_{n} \in \mathscr{R}(A)$ such that $h_{n} \leqslant f \leqslant g_{n}$ and $\int_{A}\left(g_{n}-h_{n}\right)<1 / n, n=1$, $2, \ldots$. Since $\mathscr{M}$ contains all compact subsets of $X$, we see that $g_{n}$ and $h_{n}$, and also $g=\inf g_{n}$ and $h=\sup h_{n}$, belong to $\mathscr{L}_{\mu}(A)$. We have $h \leqslant f \leqslant g$ and

$$
0 \leqslant(L) \int_{A}(g-h) d \mu=\int_{A}(g-h) \leqslant \int_{A}\left(g_{n}-h_{n}\right)<\frac{1}{n}
$$

for $n=1,2, \ldots$ (see 6.9). Thus $(L) \int_{A}(g-h) d \mu=0$, and as $\mu$ is complete, we have $f \in \mathscr{L}_{\mu}(A)$.

If $f \in \mathscr{R}(A)$ is nonnegative, then by 3.3(ii), 3.10, and the first part of the proof, we see that $f \wedge n \in \mathscr{L}_{\mu}(A)$ for $n=1,2, \ldots$, and hence, as $f \wedge n \nearrow f$, that $f \in \mathscr{L}_{\mu}(A)$.

Since $f=\frac{1}{2}[(|f|+f)-(|f|-f)]$, the general case now follows from Corollary 3.10 .

6.13. RemarK. In view of Lemma 6.6, it is clear that the completeness of $\mu$ is essential in Theorem 6.12 .

\section{The measure induced by the integral}

Let $C_{0}(X)$ denote the family of all continuous functions $f \in \mathscr{F}(X)$ for which the set $\operatorname{supp} f=\{x \in X: f(x) \neq 0\}^{-}$is compact. If $f \in C_{0}(X)$, then by 2.1(i), there is a finite disjoint collection $\mathscr{A}_{f} \subset \mathscr{A}$ such that $\operatorname{supp} f \subset\left(\cup \mathscr{A}_{f}\right)^{\circ}$. Letting 
$J(f)=\sum_{A \in \mathscr{A}_{f}} \int_{A} f$, we see easily that $J(f)$ does not depend on the choice of $\mathscr{A}_{f}$, and that $J: C_{0}(X) \rightarrow \mathbf{R}$ is a nonnegative linear functional. By the Riesz representation theorem (see [8, (13.9), page 164]), there is a unique regular, complete and saturated measure space $(X, \mathcal{N}, \nu)$ such that $J(f)=(L) \int_{X} f d \nu$ for each $f \in$ $C_{0}(X)$ (see [8, (9.1) and (8.12), pages 109 and 100]).

We show first that on compact sets, the measure $\nu$ can be calculated directly from the volume $v$.

7.1. Proposition. Let $K \subset X$ be compact. Then $\nu(K)=\inf \sum_{i=1}^{n} v\left(A_{i}\right)$ where the infimum is taken over all disjoint collections $\left\{A_{1}, \ldots, A_{n}\right\} \subset \mathscr{A}$ with $K \subset$ $\left(\mathrm{U}_{i=1}^{n} A_{i}\right)^{\circ}$.

Proof. Denote by $\alpha$ the right hand side of the desired equality, and choose a disjoint collection $\left\{A_{1}, \ldots, A_{n}\right\} \subset \mathscr{A}$ such that $G=\left(\cup_{i=1}^{n} A_{i}\right)^{\circ}$ contains $K$. There is an $f \in C_{0}(X)$ with $\chi_{K} \leqslant f \leqslant \chi_{G}$, and so

$$
\nu(K) \leqslant J(f)=\sum_{i=1}^{n} \int_{A_{1}} f \leqslant \sum_{i=1}^{n} v\left(A_{i}\right) .
$$

From the arbitrariness of $\left\{A_{1}, \ldots, A_{n}\right\}$, we obtain $\nu(K) \leqslant \alpha$.

To prove the reverse inequality, choose an open set $H$ containing $K$, and use 2.1(i) to find a disjoint collection $\left\{B_{1}, \ldots, B_{k}\right\} \subset \mathscr{A}$ such that

$$
K \subset\left(\bigcup_{i=1}^{k} B_{i}\right)^{\circ} \subset\left(\bigcup_{i=1}^{k} B_{i}\right)^{-} \subset H .
$$

Let $C=\left(\cup_{i=1}^{k} B_{i}\right)^{-}$, and find a $g \in C_{0}(X)$ with $\chi_{C} \leqslant g \leqslant \chi_{H}$. Then

$$
\alpha \leqslant \sum_{i=1}^{k} v\left(B_{i}\right) \leqslant \sum_{i=1}^{k} \int_{B_{i}} g \leqslant J(g) \leqslant \nu(H),
$$

and from the regularity of $\nu$, we obtain $\alpha \leqslant \nu(K)$.

7.2. Corollary. If $A \in \mathscr{A}$, then $\nu\left(A^{\circ}\right) \leqslant v(A) \leqslant \nu\left(A^{-}\right)$.

Proof. If $K \subset A^{\circ}$ is compact, then $\nu(K) \leqslant v(A)$ by 7.1. By the regularity of $\nu$, also $\nu\left(A^{\circ}\right) \leqslant v(A)$. If $\left\{A_{1}, \ldots, A_{n}\right\} \subset \mathscr{A}$ is a disjoint collection with $A^{-} \subset$ $\left(\cup_{i=1}^{n} A_{i}\right)^{\circ}$, then $v(A) \leqslant \sum_{i=1}^{n} v\left(A_{i}\right)$. Thus by 7.1, also $v(A) \leqslant \nu\left(A^{-}\right)$.

7.3. Proposition. $A$ set $E \subset X$ belongs to $\mathscr{N}$ if and only if $\chi_{E} \in \mathscr{R}(A)$ for each $A \in \mathscr{A}$. Moreover, if $E \in \mathscr{N}$ and $E^{-}$is compact, then $\nu(E)=\sum_{i=1}^{n} \int_{A_{i}} \chi_{E}$ for each disjoint collection $\left\{A_{1}, \ldots, A_{n}\right\} \subset \mathscr{A}$ with $E \subset\left(\bigcup_{i=1}^{n} A_{i}\right)^{\circ}$. 
Proof. Let $\left\{A_{1}, \ldots, A_{n}\right\} \subset \mathscr{A}$ be a disjoint collection, and let $Y=\left(\cup_{i=1}^{n} A_{i}\right)^{\circ}$. Further, let

$$
\Upsilon=\left\{E \subset Y: \chi_{E} \in \mathscr{R}\left(A_{i}\right), i=1, \ldots, n\right\},
$$

and for $E \in \Upsilon$ set $\tau(E)=\sum_{i=1}^{n} \int_{A_{i}} \chi_{E}$. It follows from 3.3.(i), (iii), 5.1, and 5.6 that $\Upsilon$ is a $\sigma$-algebra in $Y$ containing all open subsets of $Y$, and that $\tau$ is a finite complete measure on $\Upsilon$. From 5.6, we also obtain that $\tau(E)=\nu(E)$ whenever $E \in \Upsilon$ is open or compact. We want to prove that $\tau$ is regular in the sense of [8, (9.1), page 109]. As $\nu$ is regular in this sense, it suffices to show that $\tau(E)=$ $\inf \{\tau(G)$ : $G$ open, $E \subset G \subset Y\}$ for each $E \in \Upsilon$.

To this end, let $E \in \Upsilon$, and let $\varepsilon>0$. By 6.2, there are bounded lower semicontinuous functions $g_{i} \in \mathscr{R}\left(A_{i}\right)$ such that $g_{i} \geqslant \chi_{E} \mid A_{i}^{-}$and $\int_{A_{i}} g_{i}<\int_{A_{i}} \chi_{E}$ $+\varepsilon / n, i=1, \ldots, n$. For $x \in \bigcup_{i=1}^{n} A_{i}^{-}$, let $g(x)=\Lambda\left\{g_{i}(x): x \in A_{i}^{-}\right\}$. It is easy to see that $g$ is a bounded lower semicontinuous function on $\mathrm{U}_{i=1}^{n} A_{i}^{-}$, and $g \nmid Y \geqslant \chi_{E} \uparrow Y$. By 5.7, $g \in \mathscr{R}\left(A_{i}\right)$ for $i=1, \ldots, n$, and

$$
\sum_{i=1}^{n} \int_{A_{i}} g \leqslant \sum_{i=1}^{n} \int_{A_{i}} g_{i}<\sum_{i=1}^{n} \int_{A_{i}} \chi_{E}+\varepsilon=\tau(E)+\varepsilon .
$$

If $G=\{x \in Y: g(x)>1-\varepsilon\}$, then $G \subset Y$ is open, $E \subset G$, and $\chi_{G} \leqslant g /(1-\varepsilon)$. Thus

$$
\tau(E) \leqslant \tau(G)=\sum_{i=1}^{n} \int_{A_{i}} \chi_{G} \leqslant \frac{1}{1-\varepsilon} \sum_{i=1}^{n} \int_{A_{i}} g<\frac{1}{1-\varepsilon}[\tau(E)+\varepsilon],
$$

and the desired equality follows by the arbitrariness of $\varepsilon$.

Now by [8, (9.10), page 114], $\Upsilon=\{E \in \mathscr{N}: E \subset Y\}$ and $\tau(E)=\nu(E)$ for each $E \in \Upsilon$. It follows that the proposition holds for each $E \subset X$ with $E^{-}$compact; for by 2.1(i), such a set $E$ is contained in $\left(\cup_{i=1}^{n} A_{i}\right)^{\circ}$ for some disjoint collection $\left\{A_{1}, \ldots, A_{n}\right\} \subset \mathscr{A}$. Since for any $E \subset X$ and $A \in \mathscr{A}$ we have $\chi_{E} \in$ $\mathscr{R}(A)$ if and only if $\chi_{E \cap A^{-}} \in \mathscr{R}(A)$, the proof is completed by an application of $[8,(9.8)$, page 112].

7.4. THEOREM. The following conditions are equivalent.

(i) Let $A \in \mathscr{A}$, and let $f, g \in \mathscr{F}\left(A^{-}\right)$be such that $f(x)=g(x)$ for each $x \in A$. Then $f \in \mathscr{R}(A)$ if and only if $g \in \mathscr{R}(A)$, in which case $\int_{A} f=\int_{A} g$.

(ii) $\mathscr{A} \subset \mathscr{N}$, and $\nu(A)=v(A)$ for each $A \in \mathscr{A}$.

(iii) There is a locally Radon measure space $(X, \mathscr{M}, \mu)$ such that $\mathscr{A} \subset \mathscr{M}$ and $\mu(A)=v(A)$ for each $A \in \mathscr{A}$.

Proof. (i) $\Rightarrow$ (ii). Let $A, B \in \mathscr{A}$, and let $\left\{C_{1}, \ldots, C_{m}\right\} \subset \mathscr{A}$ be a disjoint collection such that $B-A=\bigcup_{i=1}^{m} C_{i}$. By (i) and 3.3(ii), $\chi_{A} \in \mathscr{R}(A \cap B)$ and $\int_{A \cap B} \chi_{A}=v(A \cap B)$, and also $\chi_{A} \in \mathscr{R}\left(C_{i}\right)$ and $\int_{C_{i}} \chi_{A}=0, i=1, \ldots, m$. Using 
3.6, we see that $\chi_{A} \in \mathscr{R}(B)$ and $\int_{B} \chi_{A}=v(A \cap B)$. Thus $A \in \mathscr{N}$ by Proposition 7.3. Moreover, if $\left\{B_{1}, \ldots, B_{n}\right\} \subset \mathscr{A}$ is a disjoint collection with $A \subset$ $\left(\cup_{i=1}^{n} B_{i}\right)^{\circ}$, then, again by 7.3 , we have

$$
\nu(A)=\sum_{i=1}^{n} \int_{B_{i}} \chi_{A}=\sum_{i=1}^{n} v\left(A \cap B_{i}\right)=v(A) .
$$

The implication (ii) $\Rightarrow$ (iii) is trivial, for $(X, \mathcal{N}, \mu)$ is a locally Radon measure space by [8, (9.2), page 109]. Finally, the implication (iii) $\Rightarrow$ (i) follows from 6.10 .

The next example shows that even in a very simple situation, the integral $\int_{A} f$ may depend on the values which $f$ takes on $A^{-}-A$.

7.5. Example. In the setting of Example 2.3, let $\alpha(x)=0$ for $x<1$, and $\alpha(x)=1$ for $x \geqslant 1$. Now if $A=[0,1)$, then $\int_{A} \chi_{A^{-}}=1$ by 3.3(ii). However, it is easy to see that $\int_{A} \chi_{A}=0$; for it suffices to take a gauge $U_{\delta}$ with $\delta(x)=1-x$ for each $x \in A$.

\section{Perron and variational tests of integrability}

8.1. Definition. Let $A \in \mathscr{A}$. A function $F \in \mathscr{F}\left(\mathscr{A}_{A}\right)$ is called additive if $F(B)=\sum_{i=1}^{n} F\left(B_{i}\right)$ for each $B \in \mathscr{A}_{A}$ and each division $\left\{B_{1}, \ldots, B_{m}\right\}$ of $B$. Replacing the sign $=$ by $\geqslant$ or $\leqslant$, we obtain the definition of a superadditive or subadditive function, respectively.

Let $A \in \mathscr{A}$ and $F \in \mathscr{F}\left(\mathscr{A}_{A}\right)$. In Section 6, we defined the lower derivative ${ }_{*} F$ of $F$. Here we define the upper derivative ${ }^{*} F$ of $F$ by setting ${ }^{*} F=-{ }_{*}(-F)$. Let $f \in \mathscr{F}\left(A^{-}\right)$. A superadditive function $M \in \mathscr{F}\left(\mathscr{A}_{A}\right)$ with ${ }_{*} M \geqslant f$ is called a majorant of $f$. A subadditive function $m \in \mathscr{F}\left(\mathscr{A}_{A}\right)$ with ${ }^{*} m \leqslant f$ is called a minorant of $f$.

Now we can present the Perron test of integrability. Not surprisingly, its proof is similar to that of Lemma 6.2.

8.2. TheOREM. Let $A \in \mathscr{A}$ and $f \in \mathscr{F}\left(A^{-}\right)$. Then $f \in \mathscr{R}(A)$ if and only if for each $\varepsilon>0$ there are a majorant $M$ and a minorant $m$ of $f$ such that $M(A)-m(A)$ $\leqslant \varepsilon$; in this case

$$
\int_{A} f=\inf N(A)=\sup n(A),
$$

where the infimum and supremum are taken over all majorants $N$ and minorants $n$ of $f$, respectively. 
Proor. (i) Let $f \in \mathscr{R}(A)$, and let $\varepsilon>0$. Then there is a $U \in \Gamma_{A}$ such that $\sum_{i=1}^{s}\left|f\left(y_{i}\right) v\left(B_{i}\right)-\int_{B_{i}} f\right|<\varepsilon / 2$ for each $U$-fine subpartition $\left\{\left(B_{1}, y_{1}\right), \ldots,\left(B_{s}, y_{s}\right)\right\}$ of $A$. For $B \in \mathscr{A}_{A}$, let

$$
M(B)=\sup \sigma(f, S) \text { and } m(B)=\inf \sigma(f, S),
$$

where the supremum and infimum are taken over all $U$-fine subpartitions $S=$ $\left\{\left(B_{1}, y_{1}\right), \ldots,\left(B_{s}, y_{s}\right)\right\}$ of $A$ for which $\bigcup_{i=1}^{s} B_{i}=B$. If $S$ is such a subpartition, then

$$
\left|\boldsymbol{\sigma}(f, S)-\int_{B} f\right| \leqslant \sum_{i=1}^{s}\left|f\left(y_{i}\right) v\left(B_{i}\right)-\int_{B_{i}} f\right|<\frac{\varepsilon}{2},
$$

and it follows that

$$
\left|M(B)-\int_{B} f\right| \vee\left|m(B)-\int_{B} f\right| \leqslant \frac{\varepsilon}{2} .
$$

In particular, $M$ and $m$ are finite functions on $\mathscr{A}_{A}$, and $0 \leqslant M-m \leqslant \varepsilon$. For $x \in A^{-}$, we have ${ }^{*} m(x) \leqslant f(x) \leqslant{ }_{*} M(x)$. Indeed, if $B \in \mathscr{A}_{A \cap U(x)}$, then $\{(B, x)\}$ is a $U$-fine subpartition of $A$, and hence $m(B) \leqslant f(x) v(B) \leqslant M(B)$.

Next we show that $M$ is superadditive and that $m$ is subadditive. Let $B \in \mathscr{A}_{A}$, and let $\left\{B_{1}, \ldots, B_{k}\right\}$ be a division of $B$. For $i=1, \ldots, k$, choose a $U$-fine subpartition $S_{i}=\left\{\left(C_{1}^{i}, z_{1}^{i}\right), \ldots,\left(C_{s_{i}}^{i}, z_{s_{i}}^{i}\right)\right\}$ of $A$ with $\bigcup_{j=1}^{s_{j}} C_{j}^{i}=B_{i}$. Then

$$
S=\bigcup_{i=1}^{k} S_{i}=\left\{\left(C_{j}^{i}, z_{j}^{i}\right): j=1, \ldots, s_{i} ; i=1, \ldots, k\right\}
$$

is a $U$-fine subpartition of $A$, and $\bigcup_{i=1}^{k} \bigcup_{j=1}^{s_{i}} C_{j}^{i}=B$. Hence

$$
m(B) \leqslant \sum_{i=1}^{k} \sigma\left(f, S_{i}\right) \leqslant M(B),
$$

for $\sum_{i=1}^{k} \sigma\left(f, S_{i}\right),=\sigma(f, S)$. From the arbitrariness of the $S_{i}$ 's, we obtain

$$
m(B) \leqslant \sum_{i=1}^{k} m\left(B_{i}\right) \leqslant \sum_{i=1}^{k} M\left(B_{i}\right) \leqslant M(B) .
$$

Thus $M$ and $m$ are a majorant and a minorant of $f$, respectively.

(ii) Choose an $\varepsilon>0$, and choose a majorant $M$ and a minorant $m$ of $f$ so that $M(A)-m(A)<\varepsilon$. If $x \in A^{-}$, then there is a neighborhood $U(x)$ of $x$ such that $M(B) \geqslant[f(x)-\varepsilon] v(B)$ and $m(B) \leqslant[f(x)+\varepsilon] v(B)$ for each $B \in \mathscr{A}_{A \cap U(x)}$. This follows because ${ }_{*} M(x)>f(x)-\varepsilon$ and ${ }^{*} m(x)<f(x)+\varepsilon$. The map $x \mapsto$ $U(x)$ defines a gauge $U$ on $A$, and if $\left\{\left(A_{1}, x_{1}\right), \ldots,\left(A_{k}, x_{k}\right)\right\}$ belongs to $\mathscr{P}(A, U)$, then

$$
\begin{aligned}
& m(A)-\varepsilon v(A) \leqslant \sum_{i=1}^{k}\left[m\left(A_{i}\right)-\varepsilon v\left(A_{i}\right)\right] \leqslant \sum_{i=1}^{k} f\left(x_{i}\right) v\left(A_{i}\right) \\
& \leqslant \sum_{i=1}^{k}\left[M\left(A_{i}\right)+\varepsilon v\left(A_{i}\right)\right] \leqslant M(A)+\varepsilon v(A)<m(A)+\varepsilon[1+v(A)]:
\end{aligned}
$$


It follows that $|\sigma(f, P)-\sigma(f, Q)|<\varepsilon[1+2 v(A)]$ for each $P, Q \in \mathscr{P}(A, U)$, and so $f \in \mathscr{R}(A)$ by 3.4 .

(iii) Let $f \in \mathscr{R}(A)$, and let $N$ and $n$ be a majorant and a minorant of $f$, respectively. Choose an $\alpha>0$. Proceeding as in (ii), we can find a $U \in \Gamma_{A}$ such that

$$
n(A)-\alpha v(A) \leqslant \sigma(f, P) \leqslant N(A)+\alpha v(A)
$$

for each $P \in \mathscr{P}(A, U)$. Selecting a $P \in \mathscr{P}(A, U)$ with $\left|\sigma(f, P)-\int_{A} f\right|<\alpha$, we see that

$$
n(A)-\alpha[1+v(A)]<\int_{A} f<N(A)+\alpha[1+v(A)] .
$$

From the arbitrariness of $\alpha$, we obtain $n(A) \leqslant \int_{A} f \leqslant N(A)$, and the theorem follows.

Next we give the variational test of integrability.

8.3. Theorem. Let $A \in \mathscr{A}$ and $f \in \mathscr{F}\left(A^{-}\right)$. Then $f \in \mathscr{R}(A)$ if and only if there is an additive function $F \in \mathscr{F}\left(\mathscr{A}_{A}\right)$ having the following property: given $\varepsilon>0$, we can find a superadditive function $K \in \mathscr{F}\left(\mathscr{A}_{A}\right)$ with $K(A)<\varepsilon$, and a gauge $U$ on $A$ such that $|f(x) v(B)-F(B)| \leqslant K(B)$ for each $x \in A^{-}$and each $B \in \mathscr{A}_{A \cap \cup(x)}$. Moreover, $\int_{A} f=F(A)$.

Proof. (i) Let $f \in \mathscr{R}(A)$, and let $F(B)=\int_{B} f$ for each $B \in \mathscr{A}_{A}$. By 3.6, $F \in \mathscr{F}\left(\mathscr{A}_{A}\right)$ is additive. Given $\varepsilon>0$, we use 8.2 to find a majorant $M$ and a minorant $m$ of $f$ so that $M(A)-m(A) \leqslant \varepsilon / 2$, and we set

$$
K=M-m+\frac{\varepsilon v}{2[(1+v(A))]} .
$$

Then $K \in \mathscr{F}\left(\mathscr{A}_{A}\right)$ is superadditive, and $K(A)<\varepsilon$. If $x \in A^{-}$, then there is a neighborhood $U(x)$ of $x$ such that

$$
m(B)-\frac{\varepsilon v(B)}{2[1+v(A)]} \leqslant f(x) v(B) \leqslant M(B)+\frac{\varepsilon v(B)}{2[1+v(A)]}
$$

for each $B \in \mathscr{A}_{A \cap U(x)}$. This follows because

$$
\begin{aligned}
\left(M+\frac{\varepsilon v}{2[1+v(A)]}\right)(x) \geqslant{ }_{*} M(x)+\frac{\varepsilon}{2[1+v(A)]} & \\
& >f(x)>{ }^{*} m(x)-\frac{\varepsilon}{2[1+v(A)]} \geqslant{ }^{*}\left(m-\frac{\varepsilon v}{2[1+v(A)]}\right)(x) .
\end{aligned}
$$

Since $m \leqslant F \leqslant M$ by 8.2 , we have $|f(x) v(B)-F(B)| \leqslant K(B)$ for each $B \in$ $\mathscr{A}_{A \cap U(x)}$, and hence $x \mapsto U(x)$ is the desired gauge on $A$. 
(ii) Let $F \in \mathscr{F}\left(\mathscr{A}_{A}\right)$ be an additive function which satisfies the condition of the theorem. Choose an $\varepsilon>0$, and corresponding to it a superadditive function $K \in \mathscr{F}\left(\mathscr{A}_{A}\right)$ and a gauge $U$ on $A$. If $P=\left\{\left(A_{1}, x_{1}\right), \ldots,\left(A_{p}, x_{p}\right)\right\}$ is in $\mathscr{P}(A, U)$, then

$$
|\sigma(f, P)-F(A)| \leqslant \sum_{i=1}^{p}\left|f\left(x_{i}\right) v\left(A_{i}\right)-F\left(A_{i}\right)\right| \leqslant \sum_{i=1}^{p} K\left(A_{i}\right) \leqslant K(A)<\varepsilon,
$$

and so we see that $f \in \mathscr{R}(A)$ and $\int_{A} f=F(A)$.

8.4. Lemma. Let $A \in \mathscr{A}, f \in \mathscr{R}(A)$, and let $F(B)=\int_{B}$ f for each $B \in \mathscr{A}_{A}$. If $f$ is lower semicontinuous at $x_{0} \in A^{-}$, then ${ }_{*} F\left(x_{0}\right) \geqslant f\left(x_{0}\right)$.

Proof. Let $\alpha<f\left(x_{0}\right)$, and find a closed neighborhood $U\left(x_{0}\right)$ of $x_{0}$ such that $f(x) \geqslant \alpha$ for each $x \in U\left(x_{0}\right)$. Then $F(B) \geqslant \alpha v(B)$ for each $B \in \mathscr{A}_{A \cap U\left(x_{0}\right)}$. It follows that ${ }_{*} F\left(x_{0}\right) \geqslant \alpha$, and by the arbitrariness of $\alpha$, that ${ }_{*} F\left(x_{0}\right) \geqslant f\left(x_{0}\right)$.

8.5. Proposition. Let $A \in \mathscr{A}, f \in \mathscr{F}\left(A^{-}\right)$, and let $M$ and $m$ be a majorant and a minorant of $f$, respectively. Then $f$ has an additive majorant $N \leqslant M$, and an additive minorant $n \geqslant m$.

Proof. By 6.1 and 5.7, the functions ${ }_{*} M \wedge k, k=1,2, \ldots$, are lower semicontinuous and integrable on $A$. For $B \in \mathscr{A}_{A}$, let $N_{k}(B)=\int_{B *} M \wedge k$. Then $N_{k}$ is additive, $N_{k} \leqslant N_{k+1}$, and, since $M$ is a majorant of ${ }_{*} M \wedge k, 8.2$ implies that $N_{k} \leqslant M$. Thus $N=\lim N_{k}$ is additive, $N \leqslant M$, and, since ${ }_{*} N \geqslant{ }_{*} N_{k} \geqslant{ }_{*} M$ $\wedge k \geqslant f \wedge k$ for $k=1,2, \ldots$ (see 8.4), we also have ${ }_{*} N \geqslant f$. Since $m$ is a minorant of $f$ if and only if $-m$ is a majorant of $-f$, the proposition follows.

8.6. CoROllary. The majorant $M$ and minorant $m$ of Theorem 8.2 , as well as the function $K$ of Theorem 8.3 may be assumed to be additive.

In view of 8.5 , this is clear for $M$ and $m$. Now it suffices to observe that $K$ is a linear combination of $M, m$ and $v$ (see the proof of Theorem 8.3).

\section{References}

[1] R. Henstock, Theory of integration (Butterworths, London, 1963).

[2] R. M. McLeod, The generalized Riemann integral (MAA, Providence, 1980).

[3] E. J. McShane, 'A Riemann-type integral that includes Lebesgue-Stieltjes, Bochner and stochastic integrals', Mem. Amer. Math. Soc. 88 (1969). 
[4] E. J. McShane, 'A unified theory of integration', Amer. Math. Monthly 80 (1973), 349-359.

[5] W. F. Pfeffer, 'On the lower derivative of a set function', Canad. J. Math. 20 (1968), $1489-1498$.

[6] W. F. Pfeffer, 'An integral in topological spaces I', J. Math. Mech. 18 (1969), 953-972.

[7] W. F. Pfeffer, 'An integral in topological spaces II', Math. Scand. 27 (1970), 77-104.

[8] W. F. Pfeffer, Integrals and measures (Dekker, New York, 1977).

[9] W. F. Pfeffer, 'The Riemann-Stieltjes approach to integration', TWISK 187 (CSIR: NRIMS, Pretoria, 1980).

Department of Mathematics

King Saud University

Riyadh 11451

Saudi Arabia
Department of Mathematics

University of California

Davis, California 95616

U.S.A. 